\title{
Satellite Image Enhancement using Wavelet-domain based on Singular Value Decomposition
}

\author{
Muhammad Aamir ${ }^{1 *}$, Ziaur Rahman ${ }^{2}$, Yi-Fei Pu ${ }^{3}$ \\ College of Computer Science, \\ Sichuan University, Chengdu, China, 610065 \\ Waheed Ahmed Abro ${ }^{4}$ \\ School of Computer Science and Engineering, \\ Southeast University, Nanjing, China, 210096
}

\author{
Kanza Gulzar ${ }^{5}$ \\ School of Big Data and Software Engineering \\ Chongqing University, Chongqing, 401331, China \\ University Institute of Information Technology, \\ PMAS Arid University, \\ Rawalpindi, 46000, Pakistan
}

\begin{abstract}
Improving the quality of satellite images has been considered an essential field of research in remote sensing and computer vision. There are currently numerous techniques and algorithms used to achieve enhanced performance. Different algorithms have been proposed to enhance the quality of satellite images. However, satellite images enhancement is considered a challenging task and may play an integral role in a wide range of applications. Having received significant attention in recent years, this manuscript proposes a methodology to enhance the resolution and contrast of satellite images. To improve the quality of satellite images, in this study, first, the resolution of an image is improved. For resolution enhancement, first, the input image is decomposed into four frequency components $(L L, L H, H L$, and $H H)$ using the stationary wavelet transform (SWT). Second, Singular value matrices (SVMs) $U_{A}$ and $V_{A}$ which contains high-frequency elements of an input image are obtained using singular value decomposition (SVD). Third, the high-frequency components $(L H, H L)$ of an input image are obtained using discrete wavelet transform (DWT) and corrected by SVMs and SWT. Next, the interpolation factor is added and the high-resolution image is obtained using inverse discrete wavelet transform (IDWT). Second, the contrast of the image is optimized. For the contrast enhancement, the image is decomposed using DWT into subbands such as $(L L, L H, H L$, and $H H)$. Next, the singular value matrix (SVM) of the $L L$ sub-band is obtained which contains the illumination information. Then, SVM is modified to enhance the contrast. Finally, the image reconstructed using the IDWT. In this paper, the results from the method above are compared with existing approaches. The proposed method achieves high performance and yields more insightful results over conventional technique.
\end{abstract}

Keywords-Satellite Images; Image Enhancement; Singular Value Decomposition (SVD); Discrete Wavelet Transforms (DWT); Stationary Wavelet Transform (SWT)

\section{INTRODUCTION}

In recent years, the demand for visual information is increasing exponentially. It has been observed that visual information such as photos, audios, videos, etc. is one of the fundamental resources of receiving data which plays an integral role in people's lives [1]. Most of the information people receive and work with is visual information, particularly audio and image based. The human brain has the capability of processing visual information efficiently. The more substantial part of the human brain is dedicated to handling visual information. In contrary, the computer systems have not the ability to interpret, sense and effectively process the visual information. The performance of the visual systems can be influenced by many factors such as viewpoint and perspective, illumination, scale, deformation, and high intraclass variations. While discussing the visual information and their importance in today's life, one cannot ignore the growing demand for the quality images in many applications such as geosciences studies [2], biomedical imagining [3], astronomy [4], geographical information systems [5], surveillance [6], and remote sensing [7]. The usage of quality images is an essential requirement to achieve robust results. Furthermore, many factors such as poor illumination, adverse weather conditions, moving object, poor camera resolutions, etc. can affect the quality of images. These factors can extensively influence the contrast and resolution (dimension) of the images.

Extending the discussion about the quality of the images, in remote sensing, the quality of satellite images is also essential. Satellite images are widely used in many applications including urban planning and management, military planning, population estimation, and disaster monitoring. The resolution (dimension) and contrast of an image considered as one of the most significant quality characteristics in satellite images. The images can be transformed to get high-quality images with high resolution and standard contrast. Therefore, to improve the quality of satellite images, the image enhancement in satellite images has been an aspiration of rigorous research for numerous years. Over the years, many techniques have been proposed to enhance the resolution and contrast of 'poorquality satellite images. Besides, these algorithms have the great ability to deal with diverse aspects of image quality, such as dark areas, noise, light distortion, texture details, colour, scale, etc. The interpolation is one the most commonly used techniques to image resolution enhancement. The interpolation is a widely adopted method to increase the number of pixels in an image [8], [9], [10]. The most commonly used interpolation techniques are bilinear interpolation, wavelet zero padding [11], bi-cubic interpolation [12] and nearest neighbor interpolation.

Furthermore, the wavelet transform also plays an incredible role to enhance the quality of the image. Many wavelet-based techniques have been developed to increase the dimension of the image. In particular, discrete wavelet transform (DWT), integer wavelet transforms (IWT), and stationary wavelet trans- 
form (SWT) are the mostly adopted techniques respectively. Moreover, as we discussed, the contrast is one of the essential quality factors in the satellite images. Besides, in satellite images one the most common problems are their low contrast [13]. Contrast improvement is the pre-processing step in different remote sensing applications, and it is considered one of the most challenging issues [14]. Contrast is determined by the difference in high and low-intensity levels of an object with other objects. The contrast enhancement is necessary to provide better interpret-ability, analysis, representation, diagnosis, and perception of information in the low contrast images [15]. Low contrast image is flat with variations in the brightness and density value such as it is hard to differentiate between their dark and light values. The low contrast of an image could be the result of many reasons such as a low-resolution camera, aliasing due to improper selection of sampling rate or poor illumination.

Moreover, the information in the low contrast images is highly concentrated over a narrow range in some areas. The data may be lost in those areas which are extremely and uniformly concentrated. Therefore, to obtain a high contrast image which represents all the information to provide better input for remote sensing applications is necessary. Over the years, researchers have put lots of efforts in this field and several methods has been presented. Among them, Histogram equalization (HE) is the most popular technique to enhance the contrast of an image. Furthermore, HE is divided into two kinds: global histogram equalization (GHE) and local histogram equalization (LHE). Both of the methods have their advantages and disadvantages. GHE is a simple and more effective method to improve the contrast of the image based on global information. GHE required a less computational cost.

On the other hand, in compare to GHE, LHE is more robust in improving the overall contrast of the image, but it needed a high computational cost. Moreover, both methods change the brightness value of the input image significantly. This change results in some unwanted artefacts, which causes the saturation in uniform areas (very bright and very dark intensities) of the processed image. Furthermore, to avoid these inadequacies, several methods have been developed [16], [17], [18], [19], [20]. On the contrary, to prevent the limitations associated in HE method to enrich the contrast of the image. Singular value decomposition-based techniques have been introduced [14], [21], [22], [23], [24], [25].

In this paper, a combined approach to enhance the quality of the image has been proposed. The method first uses the bi-cubic interpolation to improve the dimension of the lowresolution images based on SVD, SWT, and DWT. Next, the contrast of the high-resolution image is enhanced based on SVD and DWT.

The rest of the paper is organized as follows: Section II discusses the theoretical background of the study, Section III elaborates the proposed methodology in detail, Section IV presents the experimental results and analysis, Section V contains the summary of the paper, and finally Section VI describes the future work.

\section{TheORETICAL BACKGROUND}

In recent years, a great deal of effort has been invested in the development of approaches to image enchantment in satellite, aerial and natural images [30][31]. Furthermore, earlier works and initiatives that have been made to enrich the contrast and resolution of the images adequately are briefly examined as follows. Neena et al. [8], proposed a technique for image enhancement. The proposed technique up-scaled the resolution of an input image based on bi-cubic interpolation and stationary wavelet transform (SWT). The proposed model achieved excellent performance over conventional methods but required a high computational cost. Zhang et al. [9], suggested a model which combines two methods of interpolation that rational and fractal models which upscale the input image (low-resolution) to high-resolution image. The proposed method achieves robust performance with smooth edges and less blurring. However, the process produced high complexity. Kang et al. [10], introduced a model, which uses image interpolation based on direct adaption and image restoration. The proposed method adopts the bi-linear and bicubic interpolation filters to reduce the jagging artefacts in edge regions. The experimental result shows that the method achieved good interpolation efficiency compared to state-of-the-art techniques. Though, the system has some weaknesses. Tinku et al. [11], has proposed an algorithm for image resolution. The technique is based on both non-adaptive and adaptive image interpolation. The process yields robust performance and improves the quality of an image vigorously. Furthermore, Keys et al [12], has developed a method which is based on cubic convolutional interpolation. The proposed method has a robust performance in resampling the discrete data and yields the excellent performance in scaling up the resolution on an image.

Demirel et al. [21][22] have developed the model to contrast enhancement. The method used the singular value decomposition to equalized the processed image. In comparing to HE methods, the proposed methods yield good performance. However, the method performance is not good for the low contrast images as the intensity of Low-contrast image varies in three different types, i.e., low range (dark image), mid-range (grey image), and high range (bright image). The method used in Demirel et al. [21], [22], is based on the scaling of singular value and is not suitable for mid-range (grey image). Since, a correction coefficient is close to "1", which suggests that there is no significant change in a singular matrix. Hence, there is no substantial improvement in the intensity of input images. The method is only suitable for low range intensity (dark) images [8].

Contrary to Demirel et al. findings, the proposed model is ideal for both Low and mid-range intensity images (dark and grey) and carries the ability to enhance the contrast. So, based on this experimental finding, Atta et al. [23], the proposed model presents new insights. The method produces better results for the for mid-range (grey image) intensities value. However, its performance is reduced in comparison to the methods [21], [22] in term of mean, standard deviation, and entropy values.

Based on these findings, the authors are of the view that their experimental approach will provide more meaningful insights into the image quality of the satellite images through low-resolution and low-contrast satellite imaging. The current 
study is in comparison to the recent studies and distinguished in its findings.

\section{Proposed Methodology}

In this paper, an efficient model is proposed for image enhancement from low resolution and low-contrast satellite imagery. The proposed method comprises two stages, i.e., resolution enhancement and contrast enhancement. In the first stage, the input image is taken to improve dimension by using a DWT method of Fourier transform based on SWT and SVD. Then, the DWT approach is applied to the enhanced resolution image to increase the contrast. The flowchart of the proposed framework is shown in Fig. 1.

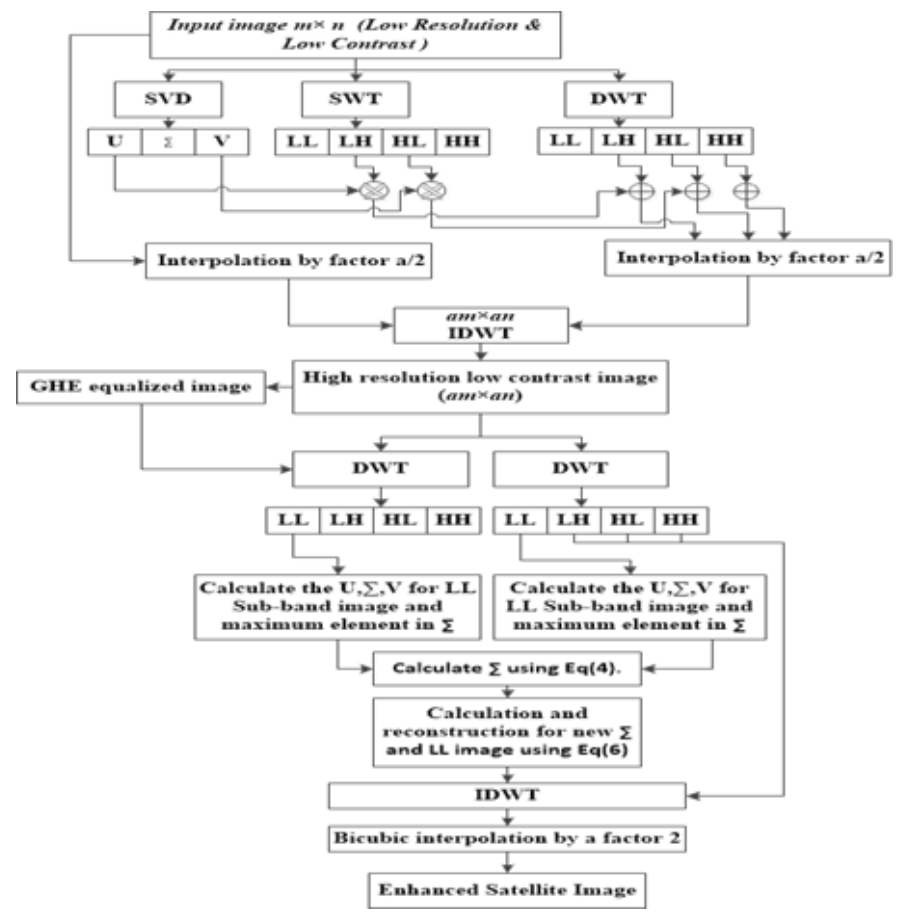

Fig. 1. Proposed method's general flow diagram.

\section{A. Image Resolution Enhancement}

In satellite images, the resolution is one of the essential features. The resolution of the images can be improved to make the system's overall performance better. In this paper, the resolution of the satellite images is increased using DWT based on SVD and SWT. First, the low-contrast input image $A$ is decomposed by applying DWT and SWT along the rows and columns of the input image separately. This decomposition results in four different sub-bands, namely, low-low $(L L)$, low-high $(L H)$, high-low $(H L)$, and high-high $(H H)$ bands, respectively.

Moreover, the edges are concentrated on $L H, H L$, and $H H$ sub-bands which are high-frequency components. Therefore, to obtain the image with high resolution, the highfrequency sub-bands $(L H, H L, H H)$ are corrected. Second, input image has converted to SVD domain to acquire the singular value matric which is the product of three matrices named an orthogonal matrix $U_{A}$, the transpose of an orthogonal matrix $V_{A}$ and a diagonal matrix $\Sigma_{A}$. The singular value matrices $U_{A}$ and $V_{A}$ contains elements with high-frequency values, and $\Sigma_{A}$ contains the illumination information such as intensity of the input image. Third, the coefficients of the orthogonal matrix $U_{A}$ along with its transpose matrix $V_{A}$ are multiplied with the high-frequency components $(L H, H L)$ of the input image obtained using SWT. Next, this multiplication result is added to high-frequency components $(L H, H L)$ obtained using DWT. Then, the bicubic interpolation with a factor of 2 is applied to an input image and estimated coefficients $(L H, H L)$ along with $H H$ of DWT. Finally, the interpolated outputs are combined using the inverse discrete wavelet transform (IDWT) to get a high dimensional image.

\section{B. Image Contrast Enhancement}

Next, the high-resolution, low contrast image $A$ and its histogram-equalized image $A$ are decomposed by applying 1-D DWT along the horizontal direction (rows) of the image and then the results are decomposed along the vertical direction (columns). The effect of the decomposition is four different kinds of sub-bands namely, low-low $(L L)$, low-high $(L H)$, high-low $(H L)$, and high-high $(H H)$ bands, as shown in Fig. 1. As we discussed in the previous section, the edges are concentrated on high-frequency $(H F)$ components such as $L H, H L$, and $H H$. Further, any transformation in lowfrequency components, such as the $L L$ sub-band, will not affect the edge information in high-frequency bands since illumination information was concentrated in the LL sub-band. Second, the $L L$ sub-band of both input image $A$ and its histogram-equalized image $A$ are chosen for further processing to improve the contrast of an image.

At this point, the decomposed image has been converted to the SVD domain to obtain the singular value matrix (SVM) which is the product of three matrices, named an orthogonal matrix $U_{A}$, the transpose of an orthogonal matrix $V_{A}$, and a diagonal matrix $\Sigma_{A}$. The SVM contains the illumination information, such as the intensity of the input image. The normalization of the SVM values is attained to change the contrast of the image. The intensity of the input image will only be affected by the modification in the singular values. The $\Sigma_{A}$ contains the intensity information of the image. It is one of the leading advantages of using SVD for image equalization [28][29]. Therefore, the SVD of an image, which can be taken as a matrix of size $N \times N$ can be defined in Eq. (1):

$$
A=U_{A} \sum_{A} V_{A}^{T}
$$

Further, the method calculates the ratio of the maximum singular value of the generated normalized matrix, with zero arithmetic mean and unity variance over a normalized image. The mathematical formulation is shown in Eq. (2):

$$
\xi=\frac{\max \left(\sum_{N(\mu=0, \mathrm{var}=1)}\right)}{\max \left(\sum_{A}\right)}
$$

Where $\sum_{N(\mu=0, \operatorname{var}=1)}$ taken as the coefficient, is the SVM of the synthetic intensity matrix and is used to regenerate an equalized image by using Eq. (3):

$$
\Xi_{e q A}=U_{A}\left(\xi \sum{ }_{A}\right) V_{A}^{T}
$$


Therefore, from the definition of SVD, the SVMs of $L L$ sub-band images of both $A$ and $\hat{A}$ are obtained. Next, the correction coefficient $\mathrm{c}$ is achieved, which is the maximum element in both SVMs and take their ratio using Eq. (4):

$$
\varsigma=\frac{\max \left(\sum_{L L_{A^{-}}}\right)}{\max \left(\sum_{L L_{A}}\right)}
$$

Where $\sum_{L L_{A^{\wedge}}}$ is the SVM of the $L L$ sub-band image of the histogram-equalized image $A$ and $\sum_{L L_{A}}$ is the SVM of the $L L$ sub-band of the input image. Eq. 5(a) and Eq. 5(b) obtain the new sub-band image:

$$
\begin{gathered}
\overline{\sum L L_{A}=}\left(\frac{\xi}{1+\xi}\right)\left(\xi \sum L L_{A}+\frac{1}{\xi} \sum L L_{A^{-}}\right) \\
L \bar{L}_{A}=U_{L L_{A}} \bar{\sum} L L_{A} V_{L L_{A}}
\end{gathered}
$$

Finally, the contrast-enhanced image $A$ is obtained by applying IDWT on the estimated $L L$ sub-band $L L_{A}$ along with high-frequency components $L H_{A}, H L_{A}$, and $H H_{A}$ subbands:

$$
\bar{A}=I D W T\left(L \bar{L}_{A}, L H_{A}, H L_{A}, H H_{A}\right)
$$

Additionally, from the achieved results, it is quite clear that the edge components of the HF bands are not disturbed and remain undamaged. The illumination information is the only thing to be manipulated alone to enhance the contrast of an image.

\section{RESUlTS AND Discussions}

In order to, check the quality and efficiency of the proposed approach. Most of the experiments were carried out on different satellite images publicly made available to use for a wide variety of remote sensing tasks, such as resolution enhancement, and contrast enhancement. Moreover, all the experiments were conducted adequately in MATLAB 2015a with the following PC specifications: $2.5-\mathrm{GHz} \mathrm{CPU}, 4 \mathrm{~GB}$ of RAM, and Windows 10. Furthermore, to evaluate the performance of the proposed technique. Four $256 \times 256$ lowcontrast with low-resolution satellite images were utilized, as shown in Fig. 2 .

Row (a) was the low resolution and contrast input image. Row (b) contains enhanced images using the proposed approach. Row (c) show enriched images using SWT-IWT methods based on SVD [8]. Row (d) illustrates the result obtained using WZP method [11]. Row (e) depicts the result attained using DWT-SWT methods [27]. Finally, Row (f) displays the image enhancement result achieved using DWT technique [26]. Moreover, the quantitative quality assessment is done to check the suitability of the proposed method. To assess the quality and to compare the performance of the proposed method with existing approaches to image enchantment. The following quality measure criterions were used such as mean square error (MSE), peak-to-signal-noise ratio (PSNR) and entropy.
These measures are the best way to evaluate performance. Mathematically, these measures were defined as:

$$
\begin{gathered}
M S E=\sqrt{\frac{\sum_{x=0}^{M-1} \sum_{y=0}^{N-1}\left\lfloor A(x, y)-A^{\prime}(x, y)\right\rfloor^{2}}{M \times N}} \\
P S N E=10 \times \log _{10}\left(\frac{R^{2}}{M S E}\right) \\
\text { Entropy }=-\sum_{i=0}^{n} p_{i} \log _{2} p_{i}
\end{gathered}
$$

Where $A(x, y)$ is the pixel value of the original image and $A^{\wedge}(x, y)$ is the pixel value of the output image $M$ is the size of the image and $R$ is the maximum pixel value.

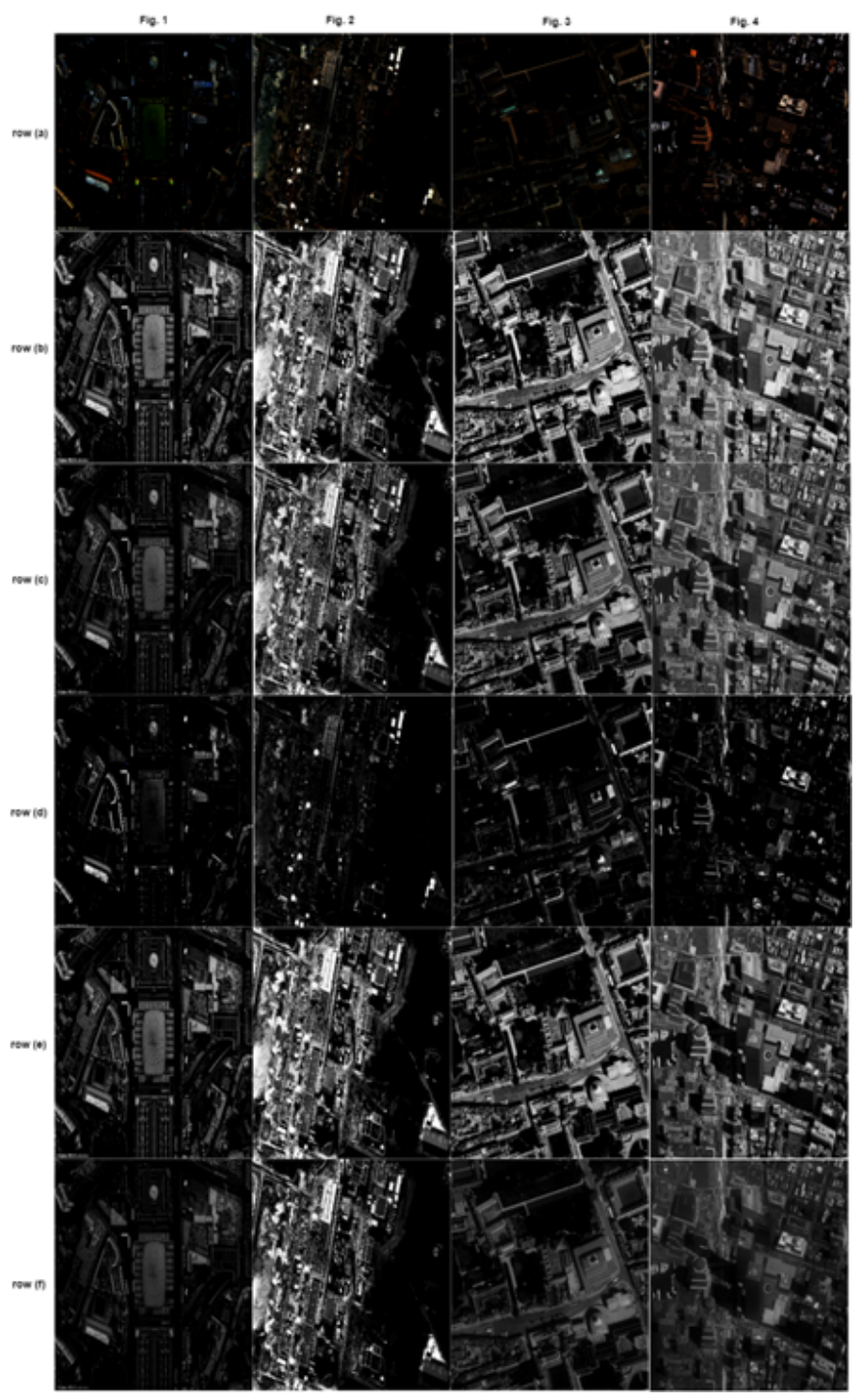

Fig. 2. Row (a) low-quality satellite images; (b) Enhanced images using the proposed approach row; (c) Enriched images using SWT-IWT methods based on SVD;(d) Improved images using WZP method;(e) Image enhancement using DWT-SWT techniques; (f) Image enhancement using DWT method. 
TABLE I. Quality Assessment Measure: Mean SQuare Error (MSE).

\begin{tabular}{llllll}
\hline Methods & Fig. 1 & Fig. 2 & Fig. 3 & Fig. 4 & Average MSE \\
& & & & & \\
\hline Tinku et al [11] & 225.21 & 253.48 & 248.81 & 205.6 & 233.75 \\
Hassan et al [26] & 98.9 & 113.71 & 109.60 & 101.13 & 105.83 \\
Demirel et al [27] & 222.10 & 251.10 & 241.94 & 199.20 & 228.58 \\
Neena et al [8] & 27.12 & 41.37 & 31.99 & 35.33 & 33.95 \\
Author's & 24.33 & 38.93 & 28.61 & 32.36 & 31.05 \\
\hline
\end{tabular}

Table I shows the comparison of the quality measure that MSE of the proposed approach with the existing methods. Four different low resolution and contrast satellite images of size $256 \times 256$ have been taken for the testing purpose. It can be seen, from Table I that the proposed method yielded a noticeable performance. As compared to existing approaches, the proposed method generated less error of $24.33 \%$. Certainty, this is due to the proposed method's image enhancing capability.

Table II. Quality Assessment Measure: Peak Signal to Noise RATIO (PSNR).

\begin{tabular}{lccccc}
\hline Methods & Fig. 1 & Fig. 2 & Fig. 3 & Fig. 4 & Average PSNR \\
\hline & & & & & \\
\hline Tinku et al [11] & 24.60 & 24.09 & 24.17 & 25.00 & 24.65 \\
Hassan et al [26] & 28.17 & 27.57 & 27.73 & 28.08 & 27.88 \\
Demirel et al [27] & 24.66 & 24.13 & 24.29 & 25.13 & 24.55 \\
Neena et al [8] & 33.79 & 31.96 & 33.08 & 32.64 & 32.86 \\
Author's & 34.26 & 32.22 & 33.56 & 33.03 & 33.26 \\
\hline
\end{tabular}

Furthermore, the comparison of the PSNR values of the proposed method produced along with other methods is given in Table II. It can be observed from the table that the recommended method results in a robust PSNR of $33.26 \mathrm{~dB}$, which is the best rate when the input images are low resolution and contrast satellite images. The proposed method dramatically improves the quality of a degraded image to an acceptable ratio which makes the technique a better approach.

TABLE III. ENTROPY: AMOUNT OF INFORMATION.

\begin{tabular}{llllll}
\hline Methods & Fig. 1 & Fig. 2 & Fig. 3 & Fig. 4 & Average Entropy \\
\hline Original Image & 8.02 & 7.91 & 7.32 & 7.45 & 7.67 \\
Tinku et al [11] & 7.31 & 6.8 & 6.71 & 7.11 & 6.98 \\
Hassan et al [26] & 7.03 & 6.89 & 6.23 & 5.66 & 6.45 \\
Demirel et al [27] & 7.82 & 7.51 & 6.69 & 7.18 & 7.3 \\
Neena et al [8] & 8.3 & 7.79 & 6.99 & 8.05 & 7.78 \\
Author's & 9.68 & 8.20 & 7.89 & 8.12 & 8.47 \\
\hline
\end{tabular}

Moreover, Table III depicts the entropy performance comparison of the proposed approach with extent methods. Furthermore, it can be analyzed from the Table III that the amount of information within an image for the proposed method is higher than prior approaches. As we can see, the proposed method achieved the excellent value of visual information that 8.47, which is considered as the best quality of a well-enhanced image.

\section{Conclusions}

In this paper, a new technique has been proposed to enhance the quality that resolution and contrast of satellite images. For resolution enhancement, the input image has been decomposed into four frequency components $(L L, L H, H L, H H)$ using SWT. Next, the SVD is applied to obtain the Singular value matrices $U_{A}$ and $V_{A}$ contains highfrequency elements. Then, the high-frequency components $(L H, H L)$ obtained using DWT are corrected by singular value matrices and SWT. Later the interpolation factor is added. Finally, the high-resolution image is obtained using IDWT. For the contrast enhancement, the proposed system enhances the contrast of high-resolution with low-contrast satellite images using the concept DWT based on SVD. The low-contrast satellite image is decomposed into DWT subbands such as $(L L, L H, H L$, andHH). Next, the SVM of the $L L$ sub-band is obtained which contains the illumination information. Then, SVM is modified to enhance the contrast. Finally, the images reconstructed using the IDWT.

In summary, the result depicts that the proposed technique achieved promising results in PSNR, entropy, and MSE. From the quantitative results, it is cleared that the application of the proposed method is useful and supports the obtained visual results better. Hence, the visual and quantitative results show that wavelet transforms work well to enhance satellite images.

\section{FUTURE WORK}

Moreover, the result obtained is sufficient and meaningful as compared to conventional approaches. Based on the results received, authors believe that the current study could be applied in many applications such as medical image processing, object recognition, surveillance, remote sensing, edge detection, video processing, feature matching, building detection in satellite images, image cropping, object detection and classification, and others. Wherever it is necessary to provide better interpretability, analysis, representation, diagnosis, and perception of information in the images, furthermore, researchers could extend this work for detecting building objects from lowcontrast satellite images.

Besides the implications as mentioned above, the authors believe that the present study could be extended for future research to enhance the quality of the satellite images which are darker and less visible and affected due to several factors such as fog or smoke, noise, color distortion, and dense weather. Apart from this, in the future, the application of fractional calculus and deep learning technologies will be introduced to enhance the quality of the images. Our next task is to make the proposed technique applicable for different size and types of images.

\section{ACKNOWLEDGMENT}

This work was supported by the National Natural Science Foundation of China under Grants 61571312, Academic and Technical Leaders Support Foundation of Sichuan province under Grants (2016)183-5, National Key Research and Development Program Foundation of China under Grants 2017YFB0802300. 


\section{REFERENCES}

[1] Ayoub, Naeem; Gao, Zhenguo; Chen, Bingcai; Jian, Muwei. 2018. "A Synthetic Fusion Rule for Salient Region Detection under the Framework of DS-Evidence Theory." Symmetry 10, no. 6: 183.

[2] Farahbakhsh, Ehsan, Rohitash Chandra, Hugo KH Olierook, Richard Scalzo, Chris Clark, Steven M. Reddy, and R. Dietmar Muller. "Computer vision-based framework for extracting geological lineaments from optical remote sensing data." arXiv preprint arXiv:1810.02320 (2018).

[3] Kavanagh DP, Gallagher MT, Kalia N. Tify: A quality-based frame selection tool for improving the output of unstable biomedical imaging. PloS one. 2019 Mar 11;14(3):e0213162.

[4] Ofek EO. A code for robust astrometric solution of astronomical images. arXiv preprint arXiv:1903.02015. 2019 Mar 5.

[5] Li X, Anderson B, Li C, Xie F. Landscape Pattern Recognition on Water Quality Protection in an Urbanizing Delta Using Remote Sensing and GIS Techniques. InRecent Developments in Intelligent Computing, Communication and Devices 2019 (pp. 899-905). Springer, Singapore.

[6] Ho GT, Tsang YP, Wu CH, Wong WH, Choy KL. A computer visionbased roadside occupation surveillance system for intelligent transport in smart cities. Sensors. 2019 Jan;19(8):1796.

[7] Gao L, Song W, Dai J, Chen Y. Road Extraction from High-Resolution Remote Sensing Imagery Using Refined Deep Residual Convolutional Neural Network. Remote Sensing. 2019 Jan;11(5):552.

[8] Neena, K. A., Aiswriya Raj, and Rajesh Cherian Roy. "Image enhancement based on stationary wavelet transform, integer wavelet transforms and singular value decomposition." International Journal of Computer Applications 58, no. 11 (2012).

[9] Zhang, Yunfeng, Qinglan Fan, Fangxun Bao, Yifang Liu, and Caiming Zhang. "Single-Image Super-Resolution Based on Rational Fractal Interpolation.” IEEE Transactions on Image Processing 27, no. 8 (2018): 3782-3797.

[10] Kang, Wonseok, Jaehwan Jeon, Eunsung Lee, Changhun Cho, Junghoon Jung, Taechan Kim, Aggelos K. Katsaggelos, and Joonki Paik. "Realtime super-resolution for digital zooming using finite kernel-based edge orientation estimation and truncated image restoration.” In Image Processing (ICIP), 2013 20th IEEE International Conference on, pp. 13111315. IEEE, 2013.

[11] Tinku Acharya, Pin-Sing Tsai,"Computational Foundations of Image Interpolation Algorithms", ACM Ubiquity Vol.8 2007.

[12] Keys, Robert. "Cubic convolution interpolation for digital image processing." IEEE transactions on acoustics, speech, and signal processing 29, no. 6 (1981): 1153-1160.

[13] Atta, Randa, and Mohammad Ghanbari. "Low-contrast satellite images enhancement using discrete cosine transform pyramid and singular value decomposition." IET Image processing 7, no. 5 (2013): 472-483.

[14] Aamir, Muhammad, Yi-Fei Pu, Ziaur Rahman, Muhammad Tahir, Hamad Naeem, and Qiang Dai. "A Framework for Automatic Building Detection from Low-Contrast Satellite Images." Symmetry 11, no. 1 (2019): 3 .

[15] Rahman, Ziaur, Yi-Fei Pu, Muhammad Aamir, and Farhan Ullah. "A framework for fast automatic image cropping based on deep saliency map detection and gaussian filter." International Journal of Computers and Applications (2018): 1-1.
[16] Kim, Y.-T.: 'Contrast enhancement using brightness preserving bihistogram equalization', IEEE Trans. Consum. Electron., 1997, 43, (1), pp. $1-8$.

[17] Ibrahim, H., Kong, N.S.P.: 'Brightness preserving dynamic histogram equalization for image contrast enhancement', IEEE Trans. Consum. Electron., 2007, 53, (4), pp. 1752-1758.

[18] Sun, C.C., Ruan, S.J., Shie, M.C., Pai, T.W.: 'Dynamic contrast enhancement based on histogram specification', IEEE Trans. Consum. Electron., 2005, 51, (4), pp. 1300-1305.

[19] Agaian, S.S., Silver, B., Panetta, K.A.: 'Transform coefficienhistogrambased image enhancement algorithms using contrastentropy', IEEE Trans. Image Process., 2007, 16, (3), pp. 741-758.

[20] Arici, T., Dikbas, S., Altunbasak, Y.: 'A histogram modification framework and its application for image contrast enhancement', IEEETrans. Image Process., 2009, 18, (9), pp. 1921-1935.

[21] Demirel, H., Anbarjafari, G., Jahromi, M.N.S.: 'Image equalization based on singular value decomposition'. Proc. 23rd IEEE Int. Symp. Computer and Information Science, Istanbul, Turkey, October 2008, pp. $1-5$.

[22] Demirel, H., Ozcinar, C., Anbarjafari, G.: 'Satellite image contrast enhancement using discrete wavelet transform and singular value decomposition', IEEE Geosci. Remote Sens. Lett., 2010, 7, (2), pp. 333-337.

[23] Atta, R., and Abdel-Kader, R.F., 2015, "Brightness preserving based on singular value decomposition for image contrast enhancement," Elsevier, Optik 126, pp. 799-803.

[24] Bhandari, A.K., Kumar, A., Padhy, P.K.: 'Enhancement of low contrast satellite images using discrete cosine transform and singular value decomposition', World Acad. Sci. Eng. Technol., 2011, 55, pp. 35-41.

[25] Kumar, A., Bhandari, A.K., Padhy, P.: 'Improved normalised difference vegetation index method based on discrete cosine transform and singular value decomposition for satellite image processing', IET Signal Process., 2012, 6, (7), pp. 617-625.

[26] Hasan Demirel and Gholamreza Anbarjafari, "Discrete Wavelet Transform-Based Satellite Image Resolution Enhancement", IEEE transactions on geosciences and remote sensing, June 2011.

[27] Hasan Demirel and Gholamreza Anbarjafari, "Image Resolution Enhancement by Using Discrete and Stationary Wavelet Decomposition" IEEE transactions on Image Processing, VOL. 20, NO. 5, May2011.

[28] Lamard, M.; Daccache,W.; Cazuguel, G.; Roux, C.; Cochener, B. Use of a JPEG-2000Wavelet Compression Scheme for Content-Based Ophtalmologic Retinal Images Retrieval. In 2005 IEEE Engineering in Medicine and Biology 27th Annual Conference; IEEE, 2005; pp. 4010-4013.

[29] Demirel, H.; Anbarjafari, G. Satellite Image Resolution Enhancement Using ComplexWavelet Transform IEEE Geosci. Remote Sens. Lett. 2010, 7, 123-126, doi:10.1109/LGRS.2009.2028440.

[30] Dai, Qiang; Pu, Yi-Fei; Rahman, Ziaur; Aamir, Muhammad. 2019 "Fractional-Order Fusion Model for Low-Light Image Enhancement." Symmetry 11, no. 4: 574.

[31] Rahman, Ziaur; Aamir, Muhammad; Pu, Yi-Fei; Ullah, Farhan; Dai, Qiang. 2018. "A Smart System for Low-Light Image Enhancement with Color Constancy and Detail Manipulation in Complex Light Environments." Symmetry 10, no. 12: 718. 